ples screened, 16 faecal samples and 18 urine samples tested positive for microsporidial DNA, and were processed for sequence analysis. Based on their Tm, 6 (3 faecal samples and 3 urine samples) were suspected of being $E$. cunic- uli, 2 faecal samples $E$. intestinalis and none of the samples resulted positive for $E$. hellem. This is a new protocol for detection and identification of microsporidial DNA in rabbit faecal and urine specimens using a real-time PCR system.

\section{Demography and health of rabbits attending primary-care in practices England}

\section{Hermien Craven, Joanna Hedley, Dan O'Neill, David Church, Dave Brodbelt}

The Royal Veterinary College, Hertfordshire, UK

Rabbits are the third most popular pet in the UK, yet very limited demographic or disorder prevalence studies are published on rabbits. The current study aimed to use the VetCompass Programme to generate an evidence base to support health reforms in rabbits.

The study population included 6,357 rabbits attending 107 primary-care veterinary practices across England during 2013. A random sample of 2,065 (32.5\%) EPRs from 104 practices were analysed in detail to extract demographic and disorder data. The median age of rabbits was 3.23 years (IQR $=1.51-4.92)$ and the median weight was $2.30 \mathrm{~kg}$ (IQR $=1.77-2.90)$. The most popular breed type was the lopeared rabbit (40.4\%). Overall, $72.0 \%$ of females and $74.0 \%$ of males had been neutered. Insurance covered $12.0 \%$ of rabbits and $23.7 \%$ were microchipped. The most frequent disorders recorded were overgrown molars (prevalence 9.3\%, 95\% Cl: 8.1-10.6), perineal soiling (5.5\%, 95\% Cl: 4.5-6.5) and overgrown incisors $(5.2 \%, 95 \% \mathrm{Cl}: 4.2-6.1)$. The most commonly affected body location was the head and neck (41.9\%, 95\% Cl: 39.8-44.1), whereas the most commonly affected organ system was the integument $(20.2 \%, 95 \% \mathrm{Cl}$ : 18.5-22.0). The most common pathophysiological process was inflammation (16\%, 95\% Cl: 15.2-16.8).

Dental disorders appeared particularly common. The study highlights the unique healthcare needs of rabbits as a pet species and the importance of a tailor-made approach to improve healthcare for rabbits in England. 